\title{
Systems biology and proteomic analysis of cerebral cavernous malformation
}

\author{
Alexander R Edelmann ${ }^{1}$, Sarah Schwartz-Baxter ${ }^{2}$, Christopher F Dibble ${ }^{3}$, Warren C Byrd ${ }^{1}$, \\ Jim Carlson ${ }^{3}$, Ivandario Saldarriaga ${ }^{1}$, and Sompop Bencharit ${ }^{*}, 1,3$ \\ ${ }^{1}$ Department of Prosthodontics and the Dental Research Center, School of Dentistry, University \\ of North Carolina, Chapel Hill, NC 27599, USA \\ 2Department of Pharmacology, School of Medicine, University of North Carolina, Chapel Hill, NC \\ 27599, USA \\ ${ }^{3}$ David H. Murdock Research Institute, North Carolina Research Campus, Kannapolis, NC 28081, \\ USA
}

\begin{abstract}
Cerebral cavernous malformations (CCM) are vascular anomalies caused by mutations in genes encoding KRIT1, OSM and PDCD10 proteins causing hemorrhagic stroke. We examine proteomic change of loss of CCM gene expression. Using human umbilical vein endothelial cells, label-free differential protein expression analysis with multidimensional liquid chromatography/ tandem mass spectrometry was applied to three CCM protein knockdown cell lines and two control cell lines: ProteomeXchange identifier PXD000362. Principle component and cluster analyses were used to examine the differentially expressed proteins associated with CCM. The results from the five cell lines revealed 290 and 192 differentially expressed proteins $(\mathrm{p}<0.005$ and $\mathrm{p}<0.001$, respectively). Most commonly affected proteins were cytoskeleton-associated proteins, in particular myosin-9. Canonical genetic pathway analysis suggests that CCM may be a result of defective cell-cell interaction through dysregulation of cytoskeletal associated proteins.
\end{abstract}

Conclusion-The work explores signaling pathways that may elucidate early detection and novel therapy for CCM.

\section{Keywords}

cerebral cavernous malformation; cytoskeletal proteins; mass spectrometry; proteomics; signaling; systems biology

\footnotetext{
(C) 2014 Informa UK Ltd

*Author for correspondence: Sompop_Bencharit@dentistry.unc.edu.

Author contributions

S Schwartz-Baxter and S Bencharit designed the experiments. S Schwartz-Baxter, J Carlson performed mass spectrometry analysis. S Bencharit, S Schwartz-Baxter, AR Edelmann, J Carlson, WC Byrd, I Saldarriaga and CF Dibble analyzed data. CF Dibble performed cell biology experiments and knockdowns. S Bencharit, WC Byrd and AR Edelmann wrote the manuscript.

Financial \& competing interests disclosure

The authors have no relevant affiliations or financial involvement with any organization or entity with a financial interest in or financial conflict with the subject matter or materials discussed in the manuscript. This includes employment, consultancies, honoraria, stock ownership or options, expert testimony, grants or patents received or pending, or royalties.

No writing assistance was utilized in the production of this manuscript.
} 
Cerebral cavernous malformation (CCM) is an inherited vascular disorder predisposing individuals to hemorrhagic stroke. CCM affects about $0.5 \%$ of the US general population, with a higher prevalence in the Hispanic population [1]. CCM is usually not known to affected individuals until the clinical symptoms occur [2,3]. These symptoms usually do not manifest until the third or fourth decade of the individual's life. Ruptured CCM lesions lead to stroke, as each new CCM lesion increases the seizure rate by $2.4 \%$ per year. CCM is also associated with seizures, recurrent headaches and focal neurological defects $[2,4]$.

Therefore, it is important to be able to detect this genetic abnormality earlier in life to prevent the CCM lesions that result in major hemorrhagic stroke [5]. CCM results from the loss of function in a CCM protein due to a genetic mutation. Recently, three genes were identified associated with CCM conditions: CCM1 (krev interaction trapped 1 [KRIT1]), CCM2 (osmosensing scaffold for MEKK3 [OSM]) and CCM3 (programmed cell death 10 [PDCD10]) that lead to the formation of CCM lesions [6-11]. These three CCM genes interact to form a protein complex in the cell. Knockdown of any CCM gene leads to defective in vitro endothelial tube formation mimicking in CCM clinical lesions [12].

The CCM1 gene encodes the KRIT1 protein that interacts with the Krev-1/rap1a signaling pathway. Krev-1/rapla is part of the family of Ras GTPase enzymes and functions as a tumor suppressor [13]. The specific function of the KRIT1 protein is unknown [14]. KRIT1 is composed of three ankyrin domains and a C-terminal FERM domain. These domains are known to interact with actin. KRIT1 functionality also stems from its interaction with integrin cytoplasmic domain-associated protein (ICAP1a). KRIT1 contains NPXY motifs on its N-terminal that is required for interaction with ICAP1a. ICAP1a also interacts with $\beta 1$ integrin. It has been shown that induced expression of KRIT1 decreases the interaction of ICAP1 $\alpha$ and $\beta 1$ integrin. Therefore, KRIT1 may function as a competitive inhibitor and regulator of $\beta 1$ integrin-ICAP1a interaction [15].

The CCM2 gene encodes OSM, and has been identified as a critical regulator for endothelial cell signaling and regulation [16,17]. OSM is located on chromosome 7p13 and is composed of N-terminal (phosphotyrosine-binding domain) and a C-terminal, with no known structural motifs [11]. OSM interacts with KRIT1's NPXY motifs through its phosphotyrosine-binding domain. OSM is a required scaffold for MEKK3-mediated p38 MAPK phosphorylation in response to osmotic stress $[16,17]$. OSM also functions as a Smurf1 recruiter through a Homologous to the E6-AP Carboxyl Terminus domain. CCM2 directs Smurf1 to a particular region of the plasma membrane where it breaks down Ras homolog gene family, member A (RhoA). This is critical, as increased RhoA protein levels result in tube formation deficiencies [18].

The CCM3 gene encodes for PDCD10, a protein whose function is apoptosis through the caspase 3 pathway [19]. PDCD10 comprises 212 amino acids with an $\mathrm{N}$-terminal binding site for serine/threonine kinase 25, the oxidant stress response enzyme [20]. This regulates PDCD10 by phosphorylation [21]. This is essential for activating germinal center kinase III MST4 following oxidative stress [22]. MST4 plays a critical role in the phosphorylation of ERM proteins that protects cells from programmed cell death. Inactivation of PDCD10 impairs this phosphorylation, making it more susceptible to oxidative stress [22]. The 
binding of PDCD10 to OSM occurs independent of KRIT1/OSM interaction [23]. PDCD10 binds to specific phosphatidylinositols and interacts with phosphatidylinositol-3-kinase (PI3K). Activation of PI3K by growth factors such as VEGF is critical to angiogenesis and perhaps CCM development. The CCM complex that is formed by these three genes is impaired by a genetic mutation of any one of the aforementioned genes [23].

This study utilized a systems biology approach to examine the global proteomic changes in human umbilical vein endothelial cells (HUVEC). Systems biology approach shows novel canonical pathways related to CCM knockdowns. These specific biomarkers have the potential to serve as diagnostic and drug-therapy targets in those afflicted with CCM. This work is a descriptive and discovery study aiming to define the novel pathways in CCM development.

\section{Methods}

\section{Cell lines \& knockdown cell lines}

Cell Culture and RNAi-bEND.3, and HUVECs cells were purchased from American type culture collection. Lentiviral genespecific shRNAs in pLKO.1 backbone were obtained from the University of North Carolina, Chapel Hill Lenti-shRNA Core Facility. Lentiviral infection was performed according to the RNAi consortium protocol. The Lipofectamine 2000 system (Invitrogen) was used for transfection and cells were subsequently selected for resistance with treatment by $4 \mu \mathrm{g} / \mathrm{ml}$ puromycin. Measurement by real-time PCR with CCMspecific primers was used to ensure knockdown (Figure 1A-C). However, the commercially available antibodies were not selective enough for all CCM proteins. Experiments were conducted after 7 days of growth in selection media in order to ensure stable protein knockdown. Off target effects were controlled for by use of multiple unique shRNAs for each protein being knocked down, and an empty vector pLKO.1 construct was used as an experimental control. bEND.3 cells were maintained in 10\% FBS/DMEM and HUVECs in $3 \% \mathrm{FBS} / \mathrm{DMEM} ; 4 \mu \mathrm{g} / \mathrm{ml}$ puromycin was used to maintain shRNA selection.

\section{Differential protein expression by LC/MS/MS analysis}

Samples were analyzed using a simultaneous label-free differential protein expression approach using a Thermo LTQ Orbitrap system. The mass spectrometry (MS) data were processed using Rosetta Elucidator (Rosetta Biosoftware, Seattle, WA, USA). Data processing steps included data pre-processing (mass calibration), mass signal alignment, protein database searching and identification using Mascot (matrix science) with SwissProt human databases. Study results included data from five cell lines analyzed in triplicate (total of 15 replicates). Data processing in Elucidator was performed in multiple steps that included mass correction and mass signal alignment of detected signals, retention time alignment of detected signals and feature selection, with features representing mass signals detected within the study set. Quality control review of the aligned data indicated the mass signals were correctly aligned in the Elucidator software. 


\section{Data analysis \& differential protein determination}

When applicable, mass signals were annotated with the corresponding peptide and protein information based on the database search results using a $1 \%$ false discovery rate cutoff. For retention time alignment, mathematical adjustments are performed to time-align the same detected signals across all samples. Quality control assessment of this retention time shift indicates that all adjustments were $<3$ min.

Principal component analysis (PCA) was performed on the collected dataset to evaluate the data for outliers and sample trends. Results from the unsupervised analysis suggest that the samples differentiate by cell line based on the mass spectrometric patterns detected. An error-weighted analysis of variance was used to determine mass patterns that correlated to differentially expressed proteins between cell lines. Signals with a $\mathrm{p}<0.01$ were selected as tentative markers, grouped according to the specific protein from which they originated and summarized by protein. PCA plots demonstrating replicate reproducibility and sample differences based on detected differentially expressed proteins are presented in (Figure 1DG). The proteomic data have been deposited to the ProteomeXchange Consortium [24] via the PRoteomics IDEntifications database partner repository with the dataset identifier PXD000362.

\section{Western blot analysis}

The total protein content in the each harvested cell line was measured using a Bradford assay with bovine serum albumin as a standard. Samples in total of $40 \mathrm{mg}$ of protein per gel lane were subjected to SDS-PAGE on a 4-15\% gradient gel. The separated proteins were transferred to nitrocellulose membrane, and the membranes were probed with anti-human myosin-9 (MYH-9) monoclonal mouse antibody (Abcam, Cambridge, MA, USA) and antihuman elongation factor $1 \gamma(\mathrm{EF} 1 \mathrm{G})$ mouse monoclonal antibody (Abcam), respectively. The dilution used for the primary antibodies was 1:10,000. Horseradish peroxidase-conjugated goat polyclonal secondary antibody to mouse IgG (Abcam) was used at a 1:5000 dilution to detect MYH-9 and EF1G. Enhanced chemiluminescence (Amersham Biosciences, Inc., Pittsburgh, PA, USA) was used to visualize detected bands (Figure 2).

\section{Modeling using ingenuity pathway analysis}

We used ingenuity pathways analysis (IPA; Ingenuity Systems, CA, USA) to evaluate if any of the biological pathways or networks have any statistical significance based on proteomic data [25]. Protein accessions from each cell line (1-4; knockdown CCM1, 2, 3 and mock knockdown with $\mathrm{p}<0.005)$ were imported into IPA. Focus proteins, mapped to corresponding gene objects in the ingenuity pathways knowledgebase, found in IPA were used to generate biological networks. IPA then built the networks with $<35$ proteins. For each network, p-value (indicating the likelihood of the focus proteins in a network being found together due to chance) and canonical pathway is calculated based on the fit of each set of significant proteins [25-27]. 


\section{Results}

\section{The proteomes of CCM knockdown cell lines}

The CCM proteins were selectively inhibited using shRNA. The controls used were a wildtype HUVEC cell line and a mock shRNA (pLKO.1-empty vector). The functional deficiency resulting from cell line knockdown was determined using an in vitro tube formation assay [12]. The cell lines figures are designated cell line 1 (knockdown CCM1), cell line 2 (knockdown CCM2), cell line 3 (knockdown CCM3), cell line 4 (wild-type HUVEC) and cell line 5 (mock knockdown). Knockdown of each CCM cell line inhibited endothelial tube formation. Each CCM cell line knockdown resulted in a similar incomplete endothelial tube phenotype. However, the specific proteomic profile changes were unique among each individual CCM cell line. To compare the proteomic changes between the five cell lines, a label-free differential expression LC/MS/MS technique was used to quantify the level of protein expression relative among the five cell lines. The amount of protein was standardized to allow comparison of each cell line. The MS data were collected in five biological triplicates for each cell line ( $\mathrm{n}=15$ total), each cell line grown in triplication. Data processing in Elucidator was performed in multiple steps that included mass correction and mass signal alignment of detected signals, retention time alignment of detected signals and feature selection, with features representing mass signals detected within the study set. The MS analytical results were processed using the Rosetta Elucidator software. When applicable, mass signals were annotated with the corresponding peptide and protein information based on the database search results using a $1 \%$ false discovery rate cutoff. The results indicated that each of the five cell lines was differentiated by its MS patterns. Differential expression of each cell line was plotted and analyzed using two different $\mathrm{p}$ values ( $\mathrm{p}<0.005$ and 0.001 ) for PCA (Figure 1D \& E). PCA analysis on the dataset demonstrated cell line trends and evaluated the data for outliers. Cell line data from the Thermo LTQ Orbitrap system was processed and resulted in the detection of 511 proteins. The differentially expressed proteomic profiles are shown in the cluster heat map. The results from the five cell lines revealed 290 and 192 differentially expressed proteins ( $\mathrm{p}<$ 0.005 and 0.001 , respectively). PCA shows clear separation of the proteomic profiles from five cell lines. Cluster analysis shows differential expression of each protein (Figure 1F \& $\mathrm{G})$. The expression of proteins including MYH-9 and EF1G were detected using western blot analysis (Figure 2). Most signaling pathways presented here have been shown to be related to CCM. We chose to only present the MYH-9 and EF1G data in this manuscript, since it had not been previously presented.

\section{Canonical pathway \& functional analysis}

From the differentially expressed proteins (analysis of variance; $\mathrm{p}<0.005$ ), we created a ratio for each protein from each CCM cell line knockdown: CCM1, CCM2, CCM3 and mock with respect to wild type using IPA. The fold change information, up and downregulation, was added based on that ratio calculation. An evaluation of the biological functions and diseases between the differentially expressed proteins and wild type was made Figures 3-5; Supplementary Figures $1 \& 2$ (supplementary material can be found online at www.informahealthcare.com/suppl/10.1586/14789450.2014.896742). This evaluation included signaling pathways controlling protein translation (EIF2, elF4/p70S6K), 
angiogenesis (mTOR, PI3K/AKT, 14-3-3 protein and VEGF), cell junctions (remodeling of epithelial adherens junctions, epithelial adherens junction, tight junction), actin (actin cytoskeleton, regulation of actin-based mobility by Rho) and others (protein ubiquitination, hypoxia in cardiovascular system, calcium, etc.). We used IPA to examine important biological functions (Supplementary Figures 3-5) including cellular growth and proliferation, cell death and survival, hematological disease, inflammatory disease, inflammatory response, cellular movement, cellular development, cellular assembly and organization, cell-to-cell signaling and interaction and cellular function and maintenance.

\section{Discussion}

The CCM cell line knockdowns led to a similar in vitro phenotype that is believed to mirror the CCM lesion in the brain [12]. The proteomic results show several common signaling pathways are significant in CCM and indicate that a separate signaling pathway for each CCM gene is present as incomplete endothelial tube formation is exhibited by the inhibition of each CCM gene. Furthermore, the specific proteomic profile changes are unique among each individual CCM cell line. The proteomic profile changes of the HUVEC cell lines were explored further using a western blot analysis to confirm the protein expression identified by MS. This supported the label-free differential protein expression analysis that used multidimensional liquid chromatography/tandem mass spectrometry (2D-LC-MS/MS) that explored the comprehensive proteomic profile changes due to cell line knockdown. The Thermo Orbitrap proteomic analysis of the data includes nine proteins known to interact with the CCM complex that were differentially expressed among the five cell lines [28]. There are proteomic changes in the majority of proteins interacting with actin and tubulin, including elongation factor $1 \mathrm{a}, \mathrm{EF} 1 \mathrm{G}, \mathrm{T}$ complex protein 1 subunit $\beta$, transgelin, cofilin and MYH-9 (Table 1) [23,28-37]. It is important to note here that MYH-9 has not been reported associated with CCM condition.

In addition to proteomic analysis, we used a systems biology approach to determine signaling pathways related to the knockdown of CCM genes. Through the use of IPA we compared each knockdown with the wild type and used the mock knockdown as our negative control. IPA demonstrates the effects of knockdown as we can see the increased signaling in EFIF2 and 14-3-3-mediated signaling (Supplementary Figure 1). These signaling pathways suggest translational disruption even with the mock knockdown. Beside the effects of the knockdown, we found four prominent groups of signaling pathways including VEGF, actin, cell junction and CCM2/OSM-related pathways. The signaling pathways are summarized in Figure 6.

The PI3K/AKT and VEGF signaling pathways (Figure 3A-C) are known to be related to CCM3/PDCD10 and CCM2/OSM [23]. Inflammation and other stress factors related to CCM [38] can activate VEGF receptors and consequently activate PI3K/AKT and possibly mTOR pathways. Activation of VEGF is perhaps a starting point for CCM-regulated signaling that controls actin stability and actin-tubulin interaction. Knockdown of CCM proteins change the expression of several actin/tubulin-related proteins (Table 1; Supplementary Table 1). Actin-related pathways including actin cytoskeleton signaling and regulation of actin-based motility by Rho (Figure 4A \& B) are potentially controlled by 
CCM complex signaling through CCM2/OSM $[12,16,17]$. The CCM complex is shown to be important in actin stability and is related to RhoA and LIM kinase signaling [12]. Knockdown of RhoA in CCM cell line knockdown reverses the CCM phenotype and restores in vitro endothelial tube formation perhaps through LIMK pathway [12]. Expression of actin-stabilizing proteins such as cofilin and transgelin, in our proteomic analysis, is also altered. Binding of cofilin and transgelin to actin stabilizes actin-tubulin complex and therefore regulates cellular process formation and cell migration [39]. Changes in cytoskeletal proteins as a result of CCM dysregulation can further disturb the cell-cell interaction as seen in several dysregulation in cell junction signal (Figure 5A-E). Disrupted cell junction and basement membrane are found in CCM lesions [40-43] and is thought to contribute to leakage and hemorrhagic stroke [44]. CCM proteins, in particular CCM2/ OSM, are known to regulate cell stress response (hypoxia signaling, ubiquination and calcium signaling) (Supplementary Figure 2). Osmotic stress and possibly other stresses may require OSM in MEKK3/MKK3 regulation of p38 activation [16]. Note that our systems biology analysis in relation to disease pathways suggest that CCM2/OSM and CCM3/PDCD10 knockdown may have more severe effects compared with CCM1/KRIT1 in hematologic diseases and inflammatory disease (Supplementary Figure 5). CCM3 knockdown has the greatest effect in inflammatory response. This may link CCM3 mutations, the rarest but most severe form of CCM, to inflammatory responses and perhaps to CCM3-mediated VEGF/mTOR/PI3K/AKT signaling. It is interesting to see that the knockdown of CCM2/OSM has the most significant effect with respect to cellular movement, cellular function and maintenance and cell growth and proliferation (Supplementary Figure 3). This may be the result of direct interaction between OSM and actin. Also, knockdown of CCM2 and CCM3 has the greatest effect on cell death and survival (Supplementary Figure 4). PDCD10 is known for its apoptotic function. This again suggests that perhaps OSM functions more closely to PDCD10 than KRIT1. We previously suggested that OSM has direct interactions with KRIT1 and PDCD10. However, there is no known direct interaction between PDCD10 and KRIT1 [23].

\section{Conclusion}

Genetic mutations of any of the CCM genes resulted in a similar incomplete in vitro endothelial tube formation and CCM clinical lesions. The proteomic profile for each cell line knockdown was unique; however, the most differentially expressed proteins were cytoskeleton-associated proteins. Our analysis suggests that these proteins, in particular MYH-9, may link CCM genes to dysregulation of actin-tubulin stability, and in turn cellcell interaction leading to CCM lesions. Our systems biology analyses demonstrate the potential role of CCM proteins in signaling pathways linking the VEGF signaling, actinmediated signaling, cell function and cell-cell interaction. However, the preliminary data will require future functional study of the proteins in each signaling pathway to definitively answer the role of CCM proteins in endothelial differentiation and CCM development. We also recognize that the limitation of our in vitro system may alter the cellular signaling. This further supports the need for future confirmative study of these proteins in cells and in animal models. In the future, CCM-related proteins may be utilized as biomarkers for CCM or used to reverse engineering the CCM signaling pathway for therapy purposes. 


\section{Supplementary Material}

Refer to Web version on PubMed Central for supplementary material.

\section{Acknowledgments}

S Bencharit was supported by the National Heart, Lung and Blood Institute NHLBI, NIH Grant R21HL092338 and the University of North Carolina at Chapel Hill Junior Faculty Development Award. The authors thank GL Johnson and his laboratory for providing experimental assistance. The authors also thank K Knagge and the PRIDE team in the assistance for the data deposition [45].

\section{References}

1. Zhang J, Clatterbuck RE, Rigamonti D, Dietz HC. Mutations in KRIT1 in familial cerebral cavernous malformations. Neurosurgery. 2000; 46(5):1272-1277. discussion 1277-1279. [PubMed: 10807272]

2. Rigamonti D, Hadley MN, Drayer BP, et al. Cerebral cavernous malformations. Incidence and familial occurrence. N Engl J Med. 1988; 319(6):343-347. [PubMed: 3393196]

3. Russell, DS.; Rubinstein, LJ. Pathology of tumours of the nervous system. Baltimore, MD, USA: Williams \& Wilkins; 1989.

4. Robinson JR, Awad IA, Little JR. Natural history of the cavernous angioma. J Neurosurg. 1991; 75(5):709-714. [PubMed: 1919692]

5. Zabramski JM, Henn JS, Coons S. Pathology of cerebral vascular malformations. Neurosurg Clin N Am. 1999; 10(3):395-410. [PubMed: 10419567]

6. Bergametti F, Denier C, Labauge P, et al. Mutations within the programmed cell death 10 gene cause cerebral cavernous malformations. Am J Hum Genet. 2005; 76(1):42-51. [PubMed: 15543491]

7. Craig HD, Gunel M, Cepeda O, et al. Multilocus linkage identifies two new loci for a Mendelian form of stroke, cerebral cavernous malformation, at 7p15-13 and 3q25.2-27. Hum Mol Genet. 1998; 7(12):1851-1858. [PubMed: 9811928]

8. Dubovsky J, Zabramski JM, Kurth J, et al. A gene responsible for cavernous malformations of the brain maps to chromosome 7q. Hum Mol Genet. 1995; 4(3):453-458. [PubMed: 7795602]

9. Dupre N, Verlaan DJ, Hand CK, et al. Linkage to the CCM2 locus and genetic heterogeneity in familial cerebral cavernous malformation. Can J Neurol Sci. 2003; 30(2):122-128. [PubMed: 12774951]

10. Guclu B, Ozturk AK, Pricola KL, et al. Mutations in apoptosis-related gene, PDCD10, cause cerebral cavernous malformation 3. Neurosurgery. 2005; 57(5):1008-1013. [PubMed: 16284570]

11. Liquori CL, Berg MJ, Siegel AM, et al. Mutations in a gene encoding a novel protein containing a phosphotyrosine-binding domain cause type 2 cerebral cavernous malformations. Am J Hum Genet. 2003; 73(6):1459-1464. [PubMed: 14624391]

12. Borikova AL, Dibble CF, Sciaky N, et al. Rho kinase inhibition rescues the endothelial cell cerebral cavernous malformation phenotype. J Biol Chem. 2010; 285(16):11760-11764. [PubMed: 20181950]

13. Sahoo T, Johnson EW, Thomas JW, et al. Mutations in the gene encoding KRIT1, a Krev-1/rap1a binding protein, cause cerebral cavernous malformations (CCM1). Hum Mol Genet. 1999; 8(12): 2325-2333. [PubMed: 10545614]

14. Serebriiskii I, Estojak J, Sonoda G, et al. Association of Krev-1/rap1a with KRIT1, a novel ankyrin repeat-containing protein encoded by a gene mapping to 7q21-22. Oncogene. 1997; 15(9):10431049. [PubMed: 9285558]

15. Zhang J, Clatterbuck RE, Rigamonti D, et al. Interaction between KRIT1 and icap1alpha infers perturbation of integrin beta1-mediated angiogenesis in the pathogenesis of cerebral cavernous malformation. Hum Mol Genet. 2001; 10(25):2953-2960. [PubMed: 11741838] 
16. Uhlik MT, Abell AN, Johnson NL, et al. Rac-MEKK3-MKK3 scaffolding for p38 MAPK activation during hyperosmotic shock. Nat Cell Biol. 2003; 5(12):1104-1110. [PubMed: 14634666]

17. Zawistowski JS, Stalheim L, Uhlik MT, et al. CCM1 and CCM2 protein interactions in cell signaling: implications for cerebral cavernous malformations pathogenesis. Hum Mol Genet. 2005; 14(17):2521-2531. [PubMed: 16037064]

18. Crose LE, Hilder TL, Sciaky N, Johnson GL. Cerebral cavernous malformation 2 protein promotes smad ubiquitin regulatory factor 1-mediated RhoA degradation in endothelial cells. J Biol Chem. 2009; 284(20):13301-13305. [PubMed: 19318350]

19. Chen L, Tanriover G, Yano H, et al. Apoptotic functions of PDCD10/CCM3, the gene mutated in cerebral cavernous malformation 3. Stroke. 2009; 40(4):1474-1481. [PubMed: 19246713]

20. Zhang H, Ma X, Deng X, et al. PDCD10 interacts with STK25 to accelerate cell apoptosis under oxidative stress. Front Biosci. 2012; 17:2295-2305.

21. Gordon J, Hwang J, Carrier KJ, et al. Protein phosphatase 2a (PP2A) binds within the oligomerization domain of striatin and regulates the phosphorylation and activation of the mammalian Ste20-Like kinase Mst3. BMC Biochem. 2011; 12:54. [PubMed: 21985334]

22. Fidalgo M, Guerrero A, Fraile M, et al. Adaptor protein cerebral cavernous malformation 3 (CCM3) mediates phosphorylation of the cytoskeletal proteins ezrin/radixin/moesin by mammalian Ste20-4 to protect cells from oxidative stress. J Biol Chem. 2012; 287(14):1155611565. [PubMed: 22291017]

23. Dibble CF, Horst JA, Malone MH, et al. Defining the functional domain of programmed cell death 10 through its interactions with phosphatidylinositol-3,4,5-trisphosphate. PLoS One. 2010; 5(7):e11740. [PubMed: 20668527]

24. ProteomeXchange. Available from: http://proteomecentral.proteomexchange.org.

25. Peddinti D, Memili E, Burgess SC. Proteomics-based systems biology modeling of bovine germinal vesicle stage oocyte and cumulus cell interaction. PLoS One. 2010; 5(6):e11240. [PubMed: 20574525]

26. Gerling IC, Singh S, Lenchik NI, et al. New data analysis and mining approaches identify unique proteome and transcriptome markers of susceptibility to autoimmune diabetes. Mol Cell Proteomics. 2006; 5(2):293-305. [PubMed: 16227630]

27. Huang Y, Yan J, Lubet R, et al. Identification of novel transcriptional networks in response to treatment with the anticarcinogen 3H-1,2-dithiole-3-thione. Physiol Genomics. 2006; 24(2):144153. [PubMed: 16317079]

28. Hilder TL, Malone MH, Bencharit S, et al. Proteomic identification of the cerebral cavernous malformation signaling complex. J Proteome Res. 2007; 6(11):4343-4355. [PubMed: 17900104]

29. Bunai F, Ando K, Ueno H, Numata O. Tetrahymena eukaryotic translation elongation factor $1 \mathrm{~A}$ (eEF1A) bundles filamentous actin through dimer formation. J Biochem. 2006; 140(3):393-399. [PubMed: 16877446]

30. Liu G, Tang J, Edmonds BT, et al. F-actin sequesters elongation factor 1alpha from interaction with aminoacyl-tRNA in a pH-dependent reaction. J Cell Biol. 1996; 135(4):953-963. [PubMed: 8922379]

31. Ejiri S. Moonlighting functions of polypeptide elongation factor 1: from actin bundling to zinc finger protein R1-associated nuclear localization. Biosci Biotechnol Biochem. 2002; 66(1):1-21. [PubMed: 11866090]

32. Seo S, Baye LM, Schulz NP, et al. BBS6, BBS10, and BBS12 form a complex with CCT/TRiC family chaperonins and mediate BBSome assembly. Proc Natl Acad Sci USA. 2010; 107(4):14881493. [PubMed: 20080638]

33. Assinder SJ, Stanton JA, Prasad PD. Transgelin: an actin-binding protein and tumour suppressor. Int J Biochem Cell Biol. 2009; 41(3):482-486. [PubMed: 18378184]

34. Keshamouni VG, Jagtap P, Michailidis G, et al. Temporal quantitative proteomics by iTRAQ 2DLC-MS/MS and corresponding mRNA expression analysis identify post-transcriptional modulation of actin-cytoskeleton regulators during TGF-beta-Induced epithelial-mesenchymal transition. J Proteome Res. 2009; 8(1):35-47. [PubMed: 19118450] 
35. Alexander JS, Minagar A, Harper M, et al. Proteomic analysis of human cerebral endothelial cells activated by multiple sclerosis serum and IFNbeta-1b. J Mol Neurosci. 2007; 32(3):169-178. [PubMed: 17873362]

36. Huang Y, Shi $\mathrm{H}$, Zhou $\mathrm{H}$, et al. The angiogenic function of nucleolin is mediated by vascular endothelial growth factor and nonmuscle myosin. Blood. 2006; 107(9):3564-3571. [PubMed: 16403913]

37. Schneider H, Errede M, Ulrich NH, et al. Impairment of tight junctions and glucose transport in endothelial cells of human cerebral cavernous malformations. J Neuropathol Exp Neurol. 2011; 70(6):417-429. [PubMed: 21572340]

38. Baev NI, Awad IA. Endothelial cell culture from human cerebral cavernous malformations. Stroke. 1998; 29(11):2426-2434. [PubMed: 9804658]

39. Olk S, Turchinovich A, Grzendowski M, et al. Proteomic analysis of astroglial connexin43 silencing uncovers a cytoskeletal platform involved in process formation and migration. Glia. 2010; 58(4):494-505. [PubMed: 19795503]

40. Glading A, Han J, Stockton RA, Ginsberg MH. KRIT-1/CCM1 is a Rap1 effector that regulates endothelial cell-cell junctions. J Cell Biol. 2007; 179(2):247-254. [PubMed: 17954608]

41. Dejana E, Tournier-Lasserve E, Weinstein BM. The control of vascular integrity by endothelial cell junctions: molecular basis and pathological implications. Dev Cell. 2009; 16(2):209-221. [PubMed: 19217423]

42. Gore AV, Lampugnani MG, Dye L, et al. Combinatorial interaction between CCM pathway genes precipitates hemorrhagic stroke. Dis Model Mech. 2008; 1(4-5):275-281. [PubMed: 19093037]

43. Whitehead KJ, Chan AC, Navankasattusas S, et al. The cerebral cavernous malformation signaling pathway promotes vascular integrity via Rho GTPases. Nat Med. 2009; 15(2):177-184. [PubMed: 19151728]

44. Gault J, Awad IA, Recksiek P, et al. Cerebral cavernous malformations: somatic mutations in vascular endothelial cells. Neurosurgery. 2009; 65(1):138-144. discussion 144-135. [PubMed: 19574835]

45. Vizcaino JA, Cote RG, Csordas A, et al. The proteomics identifications (PRIDE) database and associated tools: status in 2013. Nucleic Acids Res. 2013; 41:D1063-D1069. (Database issue). [PubMed: 23203882] 


\section{Key issues}

- Biology of cerebral cavernous malformations (CCM) lesion development is not clear.

- Proteomic global analysis of CCM gene knockdown has not been done.

- Application of label-free differential protein expression analysis to a CCM knockdown human cell line has not been reported.

- There is currently no systems biology analysis available for CCM.

- Elucidating the biology of CCM lesions may lead to a novel biomarker-based diagnosis.

- Early intervention of CCM lesions may prevent further lost of function.

- The proteins associated with specific CCM gene knockdown allow novel insight into CCM lesion development and therapy.

- Proteomic profiling and systems biology analysis may be an important tool to examine CCM in vitro and in humans. 
(D)

(A)

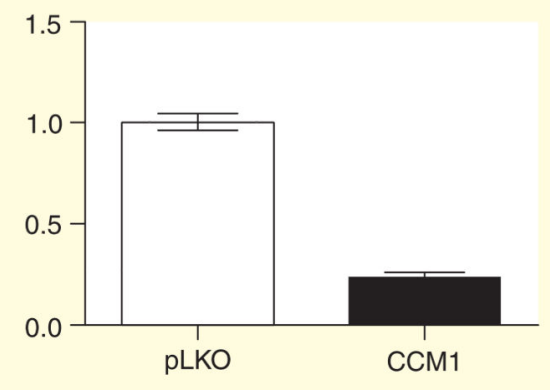

(B)

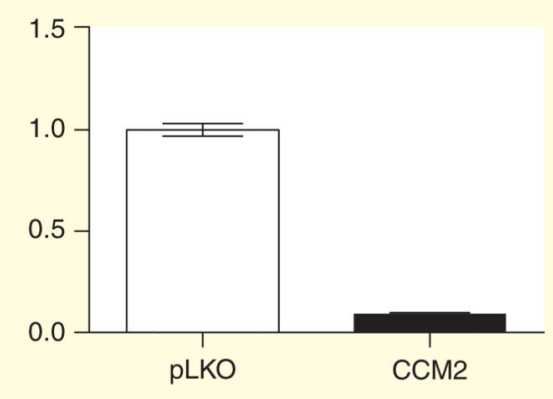

(C)

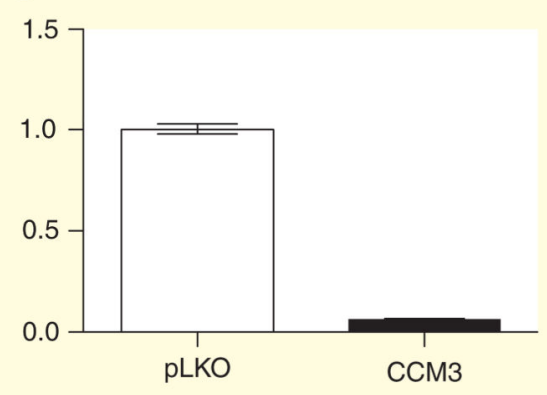

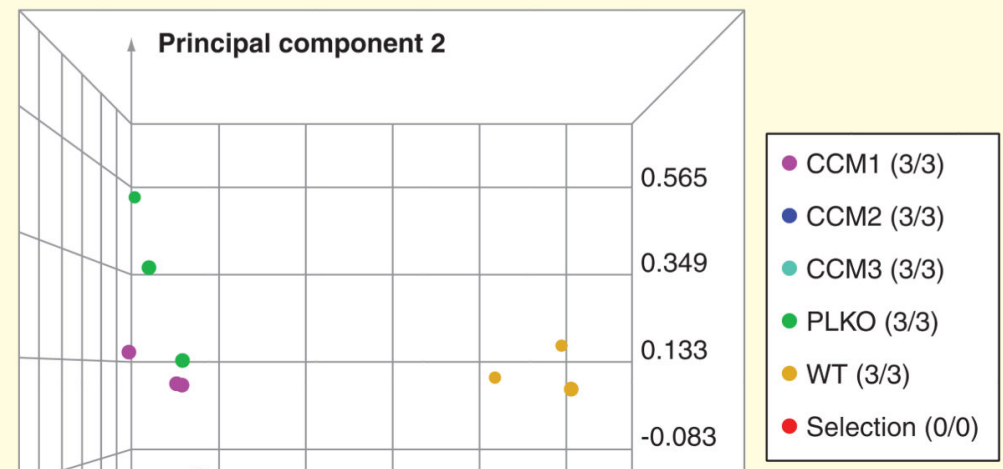

$-0.300$

Principal component 1

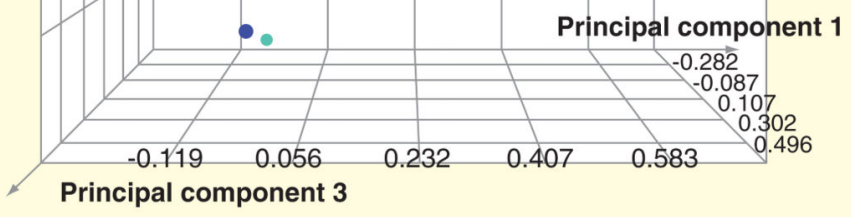

(E)

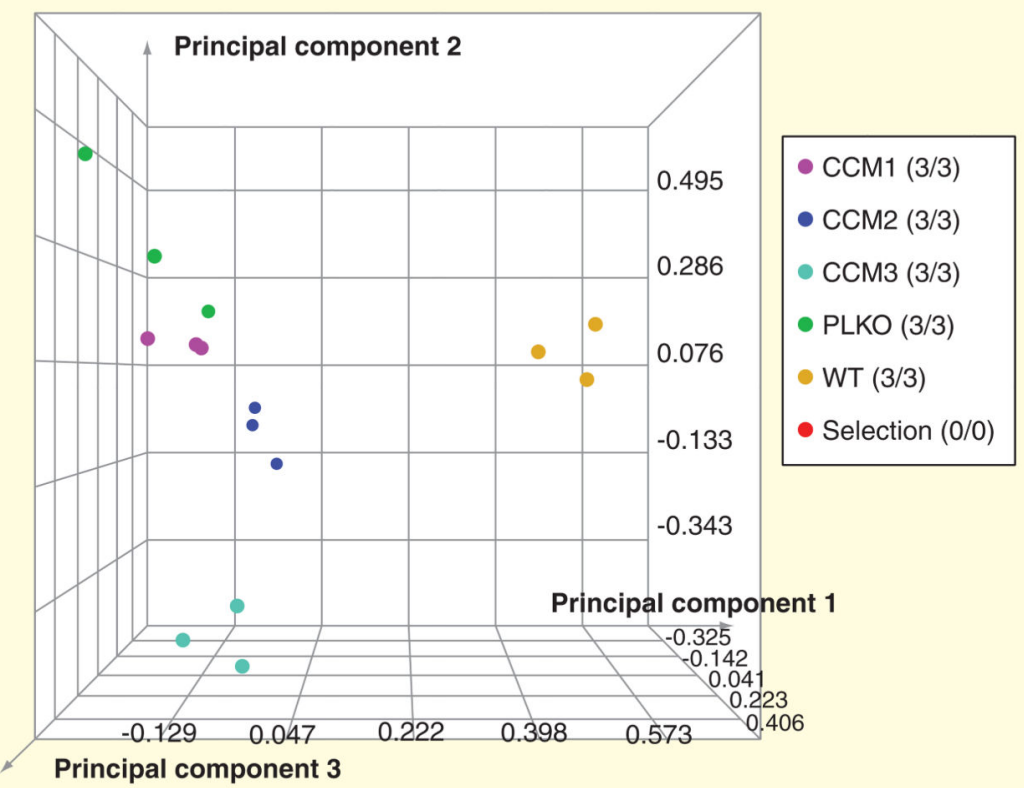



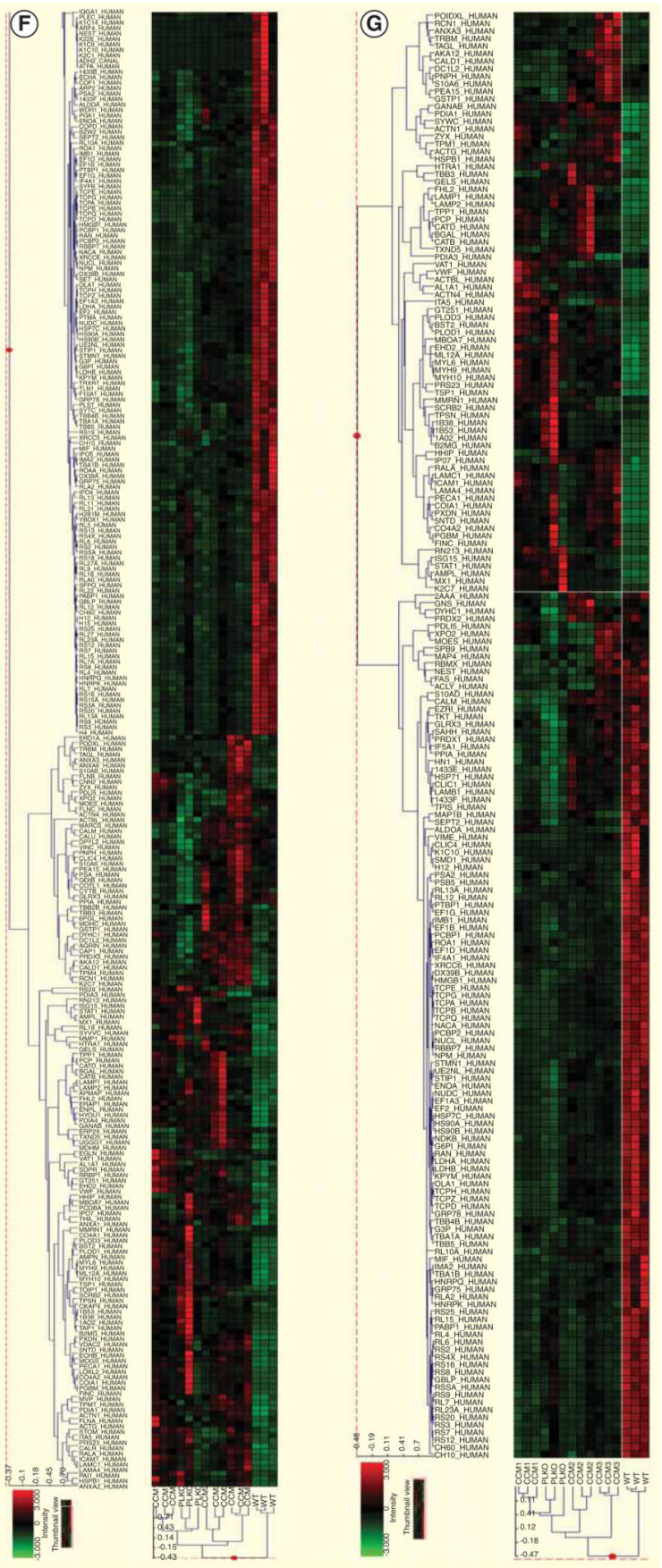

Figure 1. Proteomic analysis

(A-C) The knockdown effects of CCM1, CCM2 and CCM3 genes; (D) principal component analysis at $\mathrm{p}<0.005$; (E) principal component analysis at $\mathrm{p}<0.001 ;$ ( F) cluster analysis at $\mathrm{p}$ $<0.005$ and $(\mathbf{G})$ cluster analysis at $\mathrm{p}<0.001$.

CCM: Cerebral cavernous malformations; EF1G: Elongation factor $1 \gamma$. 


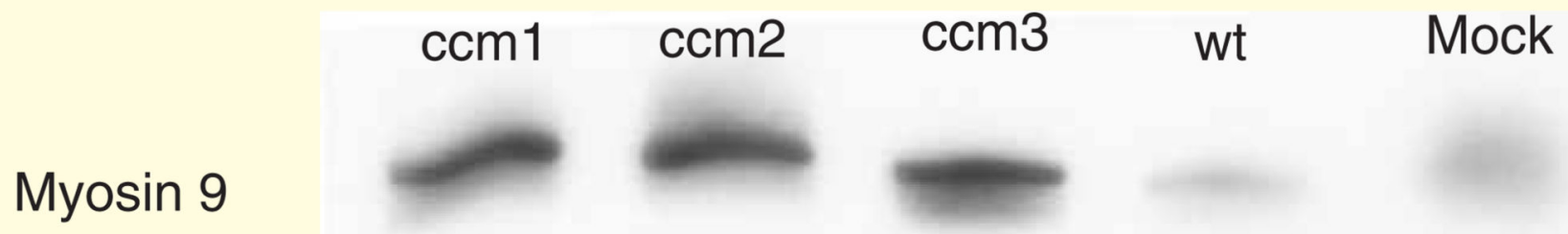

EF1G

Figure 2. Western blot analysis showing myosin-9 and elongation factor $1 \gamma$ of the knockdowns; CCM1, CCM2 and CCM3 compared with the wild-type and mock knockdown

CCM: Cerebral cavernous malformations. 
(A)

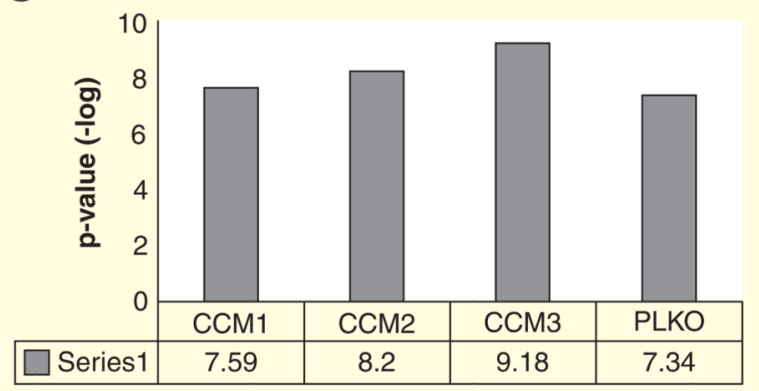

(B)

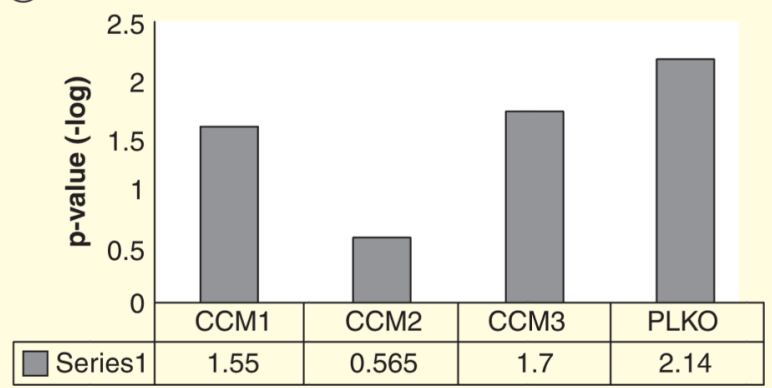

(C)

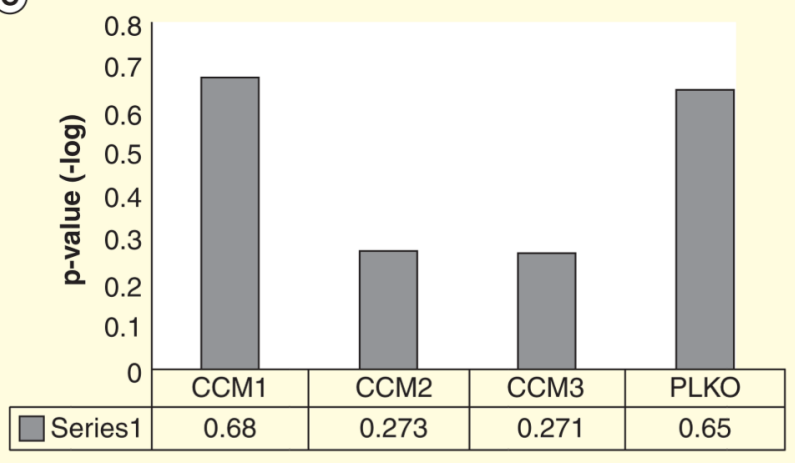

Figure 3. VEGF-related signaling based on ingenuity pathways analysis

(A) mTOR signaling; (B) P13K/AKT signaling; (C) VEGF signaling.

CCM: Cerebral cavernous malformations. 
(A)

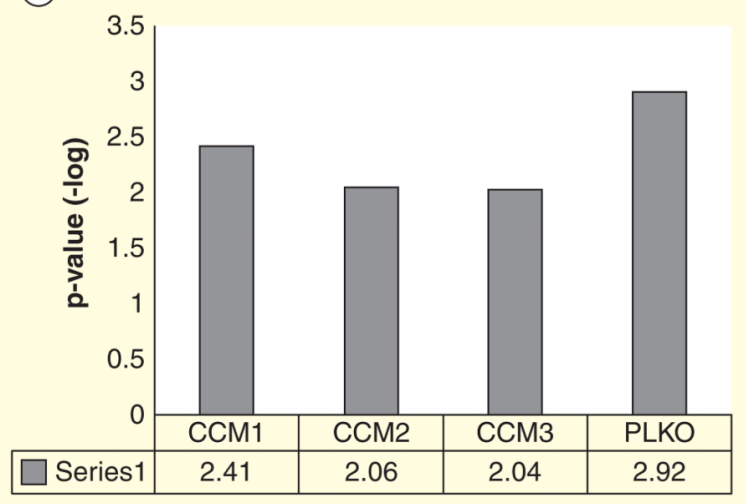

(B)

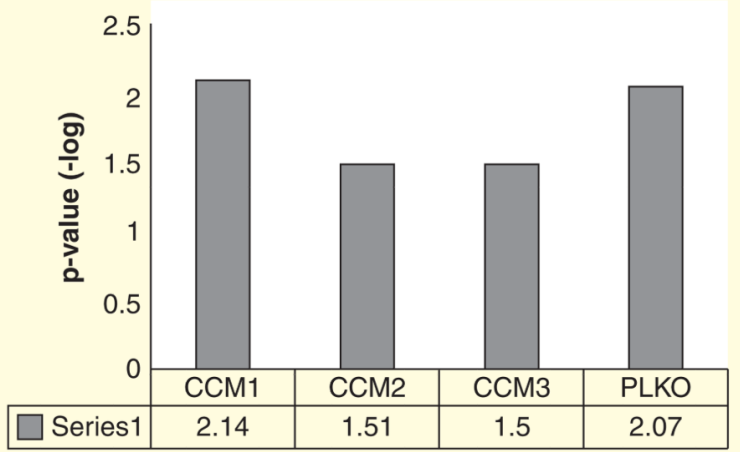

Figure 4. Actin-related signaling based on ingenuity pathways analysis

(A) Acting cytoskeleton signaling; (B) regulation of actin-based motility by Rho.

CCM: Cerebral cavernous malformations. 
(A)

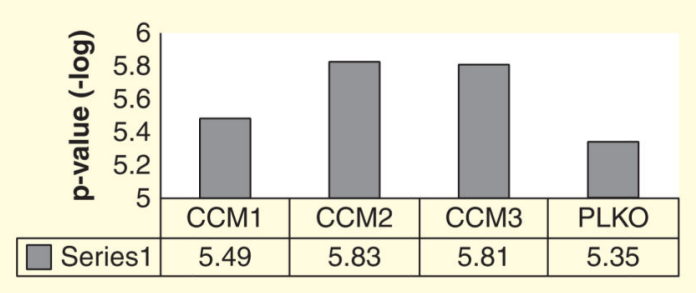

(C)

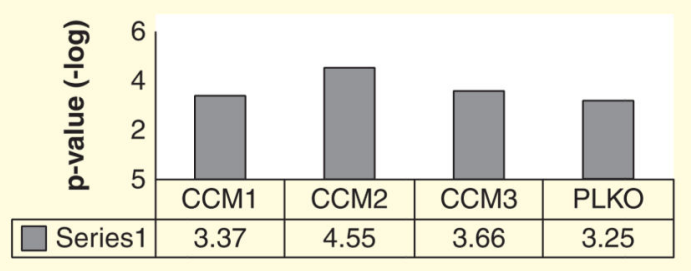

(B)

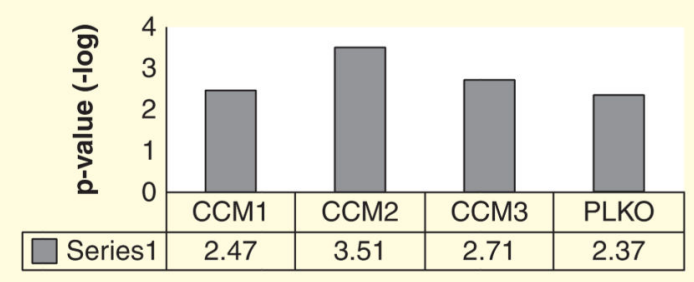

(D)

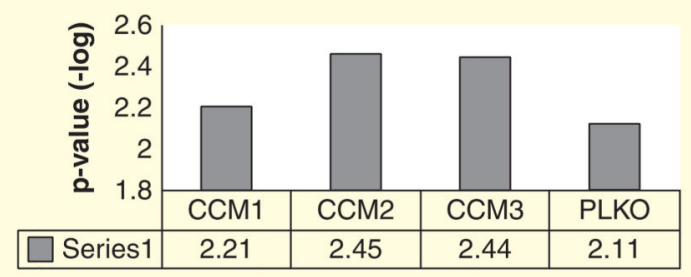

(E)

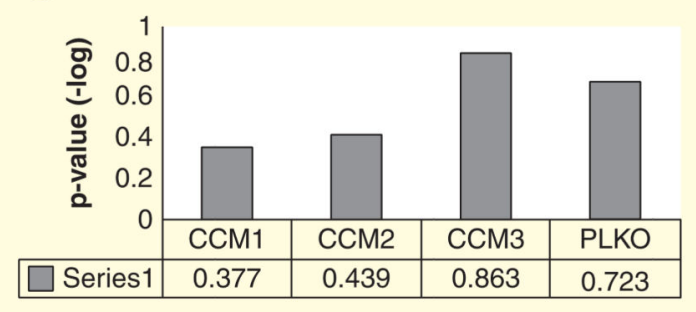

Figure 5. Epithelial junction-related signaling based on ingenuity pathways analysis

(A) Remodeling of epithelial adherens junctions; (B) germ cell-Sertoli cell junction signaling; (C) epithelial adherens junction signaling; (D) Sertoli cell-Sertoli cell junction signaling; (E) Tight junction signaling.

CCM: Cerebral cavernous malformations. 


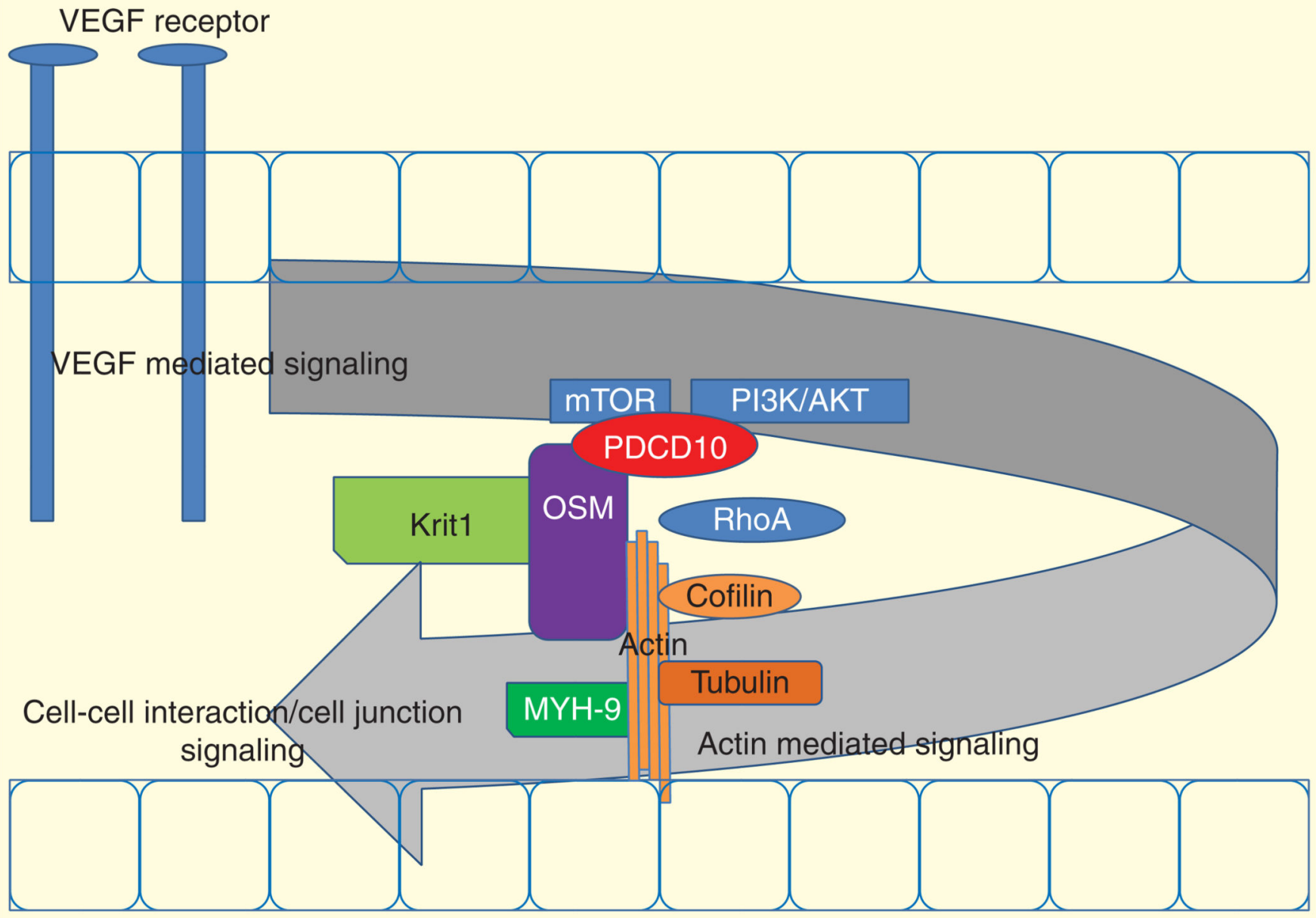

Figure 6. Proposed model of cerebral cavernous malformations development KRIT1: Krev interaction trapped 1; MYH9: Myosin-9; OSM: Osmosensing scaffold for MEKK3; PDCD10: Programmed cell death 10. 
Table 1

Selected proteins that have proteomic alteration, their functions and their relation to cerebral cavernous malformations.

\begin{tabular}{|c|c|c|}
\hline $\begin{array}{l}\text { Protein } \\
\text { name }\end{array}$ & Function & Role in CCM \\
\hline EF1a & $\begin{array}{l}\text { The } 2 \text { nd most abundant protein in the body and is } \\
\text { multifunctional. Its primary purpose is the elongation of } \\
\text { polypeptides through catalyzing GTP-dependent binding } \\
\text { of aminoacyl-tRNAs to their respective mRNA } \\
\text { anticodons [29]. It is also involved in the arrangement of } \\
\text { the actin cytoskeleton. EF1A as a translation component, } \\
\text { has a significant impact on actin- and tubulin-based } \\
\text { polymers in cells due to its abundance and relative high } \\
\left.\text { affinity ( } 1: 3 \text { molar ratio and a kd }=0.1 \mu^{2}\right) \text { [30]. It } \\
\text { associates with the actin cytoskeleton and serves as an } \\
\text { activator for phosphatidylinositol } 4 \text {-kinase. This } \\
\text { metabolite regulates phosphotidyl } 4 \text {-phosphate and } \\
\text { phosphatidylinositol } 4,5 \text { biphosphate, which regulates } \\
\text { actin filaments via capping and severing. Thus, EF1A } \\
\text { may regulate the actin cytoskeleton through the } \\
\text { phosphoinositide signaling pathway. Its importance in } \\
\text { mRNA translation has been shown in previous studies; as } \\
\text { mRNA levels for EF1A directly increase in correlation } \\
\text { with increasing amount of rapidly proliferating cells in } \\
\text { human tumors [30] }\end{array}$ & $\begin{array}{l}\text { EF1A was shown to interact directly with the CCM protein } \\
\text { complex [28]. CCM proteins such as osmosensing scaffold for } \\
\text { MEKK3 and programmed cell death } 10 \text { selectively bind to certain } \\
\text { phosphotidylinositol phosphates [23]. CCM proteins therefore can } \\
\text { regulate actin-tubulin formation through its interaction with EF1A } \\
\text { [28] as well as phosphotidylinositol phosphates [23] }\end{array}$ \\
\hline EF1G & $\begin{array}{l}\text { EF1G is a differentially expressed protein, is also a } \\
\text { subunit of the EF1 complex that is responsible for the } \\
\text { enzymatic delivery of aminoacyl-tRNAs to the ribosome. } \\
\text { The subunit's function has recently been discovered to be } \\
\text { involved in glutathione S-transferase activity [31] }\end{array}$ & $\begin{array}{l}\text { Our proteomic analysis suggested that another elongation factor, } \\
\text { while it is clear that CCM complex interacts directly with EF1A, it } \\
\text { is likely that knockdown of CCM genes also affects other EF1 } \\
\text { proteins such as EF1G [28]. However, the western blot analysis of } \\
\text { EF1G fails to show differential expression of this protein among } \\
\text { our cell lines (Figure 2). This could be explained by the limitations } \\
\text { of a western blot, which arise if the protein has been modified in } \\
\text { anyway. In addition, mass spectrometry recognizes a fragment of a } \\
\text { protein at a minute concentration, while western blot analysis } \\
\text { requires a full peptide with certain concentration }\end{array}$ \\
\hline $\begin{array}{l}\text { T complex } \\
\text { protein } 1 \\
\text { subunit } \beta\end{array}$ & $\begin{array}{l}\text { Molecular chaperone that is known to assist in the folding } \\
\text { of actin and tubulin [32]. T complex protein } 1 \gamma \text { is } \\
\text { encoded by the gene CCT3 and is also a subunit of the } \\
\text { molecular chaperone, TCP } 1 \text { complex }\end{array}$ & $\begin{array}{l}\text { It is possible that CCM knockdown disrupts normal actin-tubulin } \\
\text { interaction and in turn activates the T complex pathway. It is } \\
\text { currently unknown if CCM genes regulate the T complex directly. } \\
\text { The stability of CCM complex may also temporally stabilize the } \\
\text { actin-tubulin complex. Therefore, knockdown of a CCM gene } \\
\text { would destabilize the CCM and actin-tubulin complex and result } \\
\text { in alteration of the expression of actin-tubulin chaparones }\end{array}$ \\
\hline Transgelin & $\begin{array}{l}\text { An actin-binding protein and a tumor suppressor. It is } \\
\text { believed that it functions as a smooth cell differentiator } \\
\text { that regulates the contractile properties of smooth muscle } \\
\text { cells. In cancerous cells, expression of transgelin is } \\
\text { decreased through the activation of the Ras-MEK-ERK- } \\
\text { myc signaling pathway }\end{array}$ & $\begin{array}{l}\text { This results in a disorganized cytoskeleton of actin filaments, a key } \\
\text { trait in cancerous phenotypes. This is due to an inability of the } \\
\text { transgelin protein to cross-link with actin [33]. CCM knockdown } \\
\text { can dysregulate actin-stabilizing proteins such as cofilin. } \\
\text { Interestingly, cofilin knockdown in endothelial cells results in } \\
\text { similar alteration of transgelin expression [34] }\end{array}$ \\
\hline МYH-9 & $\begin{array}{l}\text { MYH-9 is a subunit of myosin IIA protein, the protein } \\
\text { complex has multiple roles including the maintenance of } \\
\text { cell shape }\end{array}$ & $\begin{array}{l}\text { A cytoskeleton protein had the most differential protein expression } \\
\text { among the five cell lines in both proteomic and western blot } \\
\text { analysis (Figure 2). MYH-9 protein cell line knockdown of each } \\
\text { CCM genes resulted in differential expression in comparison with } \\
\text { the two control groups (wild type and mock). Another proteomic } \\
\text { analysis has identified MYH-9 as a target in human cerebral } \\
\text { endothelial cells [35]. MYH-9 is a main regulator for endothelial } \\
\text { gap formation [36]. It is interesting to note that CCM lesions in } \\
\text { humans have defects in endothelial tight junctions, which may } \\
\text { contribute to cerebral hemorrhage [36] }\end{array}$ \\
\hline
\end{tabular}

CCM: Cerebral cavernous malformations; EF1A: Elongation factor 1a; EF1G: Elongation factor 1 $\gamma$; MYH-9: Myosin-9. 